\title{
New Developments in Imaging Cell-Based Therapy
}

\author{
Olivier Martinez ${ }^{1}$, Jane Sosabowski ${ }^{2}$, John Maher ${ }^{3-5}$, and Sophie Papa ${ }^{1,6}$ \\ ${ }^{I}$ ImmunoEngineering Group, School of Cancer and Pharmaceutical Sciences, King's Health Partners Integrated Cancer Centre, \\ Guy's Hospital, King's College London, London, United Kingdom; ${ }^{2}$ Centre for Molecular Oncology, Barts Cancer Institute, Queen \\ Mary University of London, Charterhouse Square, London, United Kingdom; ${ }^{3}$ CAR Mechanics Group, School of Cancer and \\ Pharmaceutical Sciences, King's Health Partners Integrated Cancer Centre, Guy's Hospital, King's College London, London, United \\ Kingdom; ${ }^{4}$ Department of Clinical Immunology and Allergy, King's College Hospital NHS Foundation Trust, London, United \\ Kingdom; ${ }^{5}$ Department of Immunology, Eastbourne Hospital, Eastbourne, United Kingdom; and ${ }^{6}$ Department of Medical Oncology, \\ Guy's and St. Thomas' NHS Foundation Trust, London, United Kingdom
}

Cancer immunotherapy is now established as a central therapeutic pillar in hematologic oncology. Cell-based therapies, with or without genetic modification ex vivo, have reached the clinic as the standard of care in limited indications and remain the subject of intense preclinical and translational development. Expanding on this, related therapeutic approaches are in development for solid-tumor and nonmalignant indications, broadening the scope of this technology. It has long been recognized that in vivo tracking of infused cellular therapies would provide unique opportunities to optimize their efficacy and aid in the assessment and management of toxicity. Recently, we have witnessed the introduction of novel tracers for passive labeling of cell products and advances in the introduction and use of reporter genes to enable longitudinal imaging. This review highlights the key developments over the last $5 \mathrm{y}$.

Key Words: molecular imaging; oncology; radioimmunoimaging; cell therapy; radiotracers

J Nucl Med 2019; 60:730-735

DOI: 10.2967/jnumed.118.213348

\section{H}

arnessing the immune system to eliminate established cancer has moved from the fringes of drug development to the center of cancer systemic therapy. Two broad groups of immunotherapies are dominating clinical development, namely immune checkpoint inhibitors and cell-based therapies. This review will cover developments in the imaging of cell-based therapies. Cancer-directed cell therapies may be leading the field in clinical translation, but they are closely followed by active preclinical and translational development in the fields of infection, transplantation, and autoimmunity (1). This broadening of scope is potentially transformational for medicine.

Cell-based therapies in clinical development can be divided into genetically modified therapies, consisting of either chimeric antigen receptor (CAR)- or T-cell receptor (TCR)-based cell products or nonmodified ex vivo expanded tumor-infiltrating lymphocyte therapy. Currently CAR T-cell therapy for CD19-expressing hematologic

Received Oct. 16, 2018; revision accepted Mar. 19, 2019.

For correspondence or reprints contact: Sophie Papa, Guy's Hospital, 3rd Floor, Bermondsey Wing, Great Maze Pond, London SE1 9RT, United Kingdom.

E-mail: sophie.papa@kcl.ac.uk

Published online Apr. 12, 2019.

COPYRIGHT (C 2019 by the Society of Nuclear Medicine and Molecular Imaging. cancers is leading the clinical field, with marketing authorizations granted in the United States and Europe.

CARs are membrane-spanning fusion molecules in which a targeting moiety is coupled via hinge and transmembrane elements to an activating endodomain (Fig. 1A). Genes encoding for CARs can be delivered into human T-cells via retroviral or lentiviral vectors, with the potential for coexpression of other therapeutic or imaging-related proteins. When expressed in T-cells, CARs redirect their specificity against a designated native antigen, obviating either human leukocyte antigen (HLA) expression or antigen processing. Recent spectacular results have been achieved in the treatment of

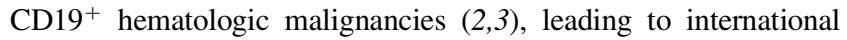
regulatory approvals. Progress in solid tumors is slower, with clinical trials not yet demonstrating the impact required for such high complexity and high-cost therapy.

TCR genetically modified cell therapies capitalize on native TCRs to target epitopes of tumor-associated antigens presented via major histocompatibility complex receptors (Fig. 1B). They benefit from more physiologic targeting and T-cell activation capability than CARs but are limited by the requirement for major histocompatibility complex presentation of tumor-associated antigen epitopes. Clinical efficacy with TCRs has been established but still requires optimization for both efficacy and safety $(4,5)$.

Clinical development of immunotherapy using ex vivo expanded tumor-infiltrating lymphocyte therapy cells has also moved forward in recent years. High response rates in melanoma single-center cohorts underpins ongoing optimism in the further development of this technology $(6,7)$.

Effective targeting of solid tumors, infections, or autoimmune disease faces multiple conceptual challenges. These include the requirement for appropriate antigen selection, optimal CAR/TCR design, successful manufacture and administration of cells, homing to sites of disease within the patient, T-cell survival, proliferation in microenvironments that are commonly immunosuppressive, and the development of immunologic memory. Toxicity of CAR T-cell therapy emerged as a significant concern at the beginning of this decade $(8-10)$. The rapid expansion in clinical experience gained through the development of the licensed CAR therapies in the past few years has led to collaborative development of evidence-based approaches to identifying and managing common toxicities (11). The additional risks of previously unidentified cross reactivity against native antigens, or lowlevel expression of target in normal tissues, has been highlighted in clinical studies $(4,9)$. This narrow therapeutic window exemplifies the importance of careful clinical translation. 


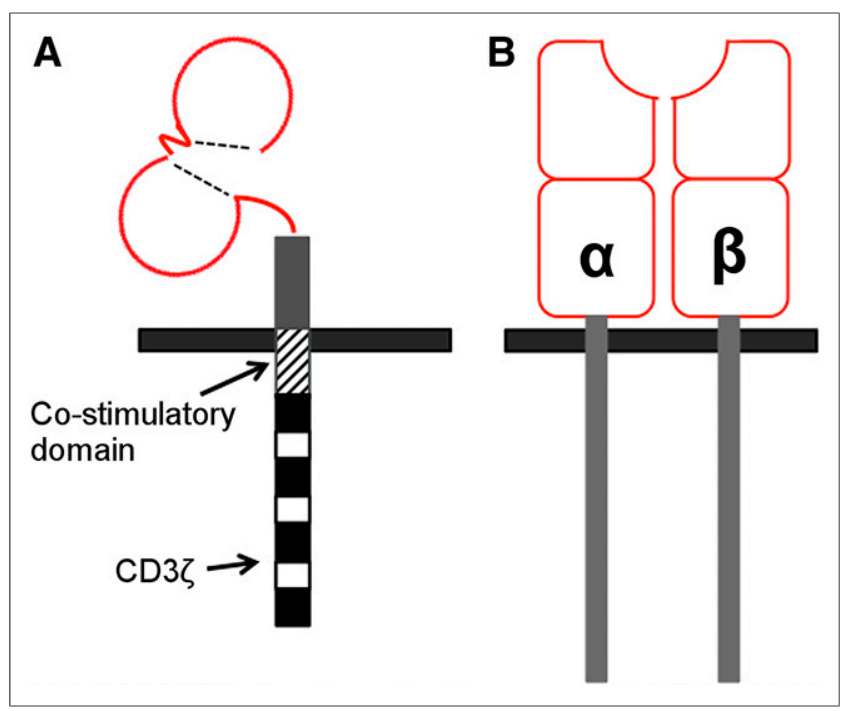

FIGURE 1. Diagram of basic CAR design (A) and TCR structure (B).

Invasive techniques requiring blood, bone marrow, and tumor sampling are routinely used in clinical trials of cell therapy. Samples can be analyzed by polymerase chain reaction, flow cytometry, and immunostaining to detect and quantify $\mathrm{T}$ cells in vivo. These approaches, although key to early clinical development of novel therapies, are inherently invasive, with the associated risk and potential lack of acceptability for patients. As cell therapy advances into the clinic in greater complexity and with expanding indications, there is a need to address safe and efficient clinical translation. Imaging approaches that enable the tracking of the adoptively transferred cells after administration to a patient should be considered an important component of translational development.

\section{EX VIVO LABELING FOR PRECLINICAL STUDIES}

Ex vivo labeling is an in vitro procedure where the contrast agents are taken up by or bound to the cells before being reinjected. It is a relatively simple method consisting of incubating cells with the imaging agent generally for a few hours. The approach originates from clinical scanning with ${ }^{111} \mathrm{In}$ - and ${ }^{99 \mathrm{~m}} \mathrm{Tc}$-pertechnetate $\left({ }^{99} \mathrm{~m}_{\mathrm{TcO}_{4}}{ }^{-}\right)$-labeled leukocytes as a means to detect sites of occult infection. Incubation time depends on the imaging agent and loading kinetics. Most of the preclinical studies involving passive labeling are done with radionuclide agents for PET and SPECT/CT imaging. However, efforts have also been made to develop imaging agents for other in vivo modalities, such as $\mathrm{CT}$ and fluorescence imaging (Fig. 2).

As the imaging tracer is not injected systemically, the ex vivo approach offers good resolution with minimal background and radioisotope load. Limitations include leakage of imaging agent as cells die, potential uptake into phagocytic cells leading to loss of specificity, an imaging window that is dictated by the half-life of the imaging tracer, and, for a proliferating cell product, dilution of signal due to lack of transmission of label to daughter cells. As these living drugs can undergo exponential expansion in vivo (100to 1,000-fold in CD19 CAR-based clinical trials), the absence of an imaging signal for ex vivo-labeled cells would not mean the absence of an expanding cell population, a critical limitation (12).

Intravital fluorescence imaging using 1,1-dioctadecyltetramethyl indotricarbocyanine iodide (DiR), a commercially available lipophilic near-infrared fluorescent dye, to label T-cells obtained from draining lymph nodes of 4T1 mammary tumor-bearing BALB/c mice represents recent progress in preclinical imaging. More than $90 \%$ of T-cells integrated DiR in their cell membrane after 30 min incubation without affecting viability and function. After adoptive transfer, the DiR-labeled T-cells accumulated at the 4T1 tumor site for up to $3 \mathrm{wk}$, which was not the case when $4 \mathrm{~T} 1$ cell lysate was coinjected, showing the specific targeting of these Tcells. Moreover, if 4T1 cells are injected after the adoptive transfer, the DiR-labeled T-cells could be imaged at the tumor site within $2 \mathrm{~h}$ (13). This approach provides medium-term imaging potential but is limited by lack of clinical utility.

Gold nanoparticles, alone or loaded with radiotracers, are also undergoing development for multimodal imaging. Using a CD19 CAR T-cell model system, $2 \times 10^{7} \mathrm{CAR}^{+}$T-cells were loaded with $1.5 \times$ $10^{12}{ }^{64} \mathrm{Cu}$-labeled gold-nanoparticles (half-life, $12.7 \mathrm{~h}$ ) by electrotransfer. After intravenous delivery of a $1.11 \times 10^{5} \mathrm{~Bq}(3-\mu \mathrm{Ci})$ dose, PET signal was recorded in the lungs after $14 \mathrm{~h}$, whereas cell-free administered nanoparticles accumulated 25-fold and 17.5-fold more in the spleen and liver, respectively. However, the electro-transfer procedure led to $50 \%$ T-cell death after $12 \mathrm{~h}$ (14). The same team then developed a multimodal (PET/MRI) contrast agent (superparamagnetic iron oxide nanoparticles [SPIO]— ${ }^{64} \mathrm{Cu}$ ) and demonstrated internalization of these positively charged nanoparticles in the same CAR ${ }^{+} \mathrm{T}$-cell system, when incubated with dimethylsulfoxide. This loading approach increased the T-cell viability to $80 \%$ after $4 \mathrm{~d}$ when $2.22 \times$ $10^{4} \mathrm{~Bq}(0.6 \mu \mathrm{Ci})$ was loaded into $1 \times 10^{8} \mathrm{CAR}^{+} \mathrm{T}$-cells. However, no in vivo data with this compound have been published (15).

Meir et al. loaded glucose-coated gold nanoparticles onto TCR transduced $\mathrm{T}$ cells specific for a melanoma tumor-associated antigen. When a limited passive loading time was applied, no significant detrimental effect was observed on interferon $\gamma$ production or proliferation of TCR transduced T cells after in vitro tumor cell coculture. Adoptive transfer into human melanoma xenograft-bearing mice showed a maximal accumulation of these cells at the tumor site after $48 \mathrm{~h}$ ( $2.3 \%$ of initial dose) by CT. The tumor signal was lost by day 5 . After $3 \mathrm{wk}$, only insignificant amounts of gold nanoparticles could be detected (16).

${ }^{111}$ In-oxine represents the gold standard for ex vivo passive labeling of cells due to its relatively long half-life $(2.8 \mathrm{~d})$ and ease of labeling procedure. To allow PET imaging at these longer time points, Charoenphun at al. developed an ${ }^{89} \mathrm{Zr}$-oxine PET probe with a half-life of $3.27 \mathrm{~d}$. This probe showed similar uptake to and better retention than ${ }^{111}$ In-oxine in 4 different cell types, including human leukocytes. An in vivo comparative study between these 2 compounds in a mouse myeloma model showed higher signal in target organs (liver, spleen, and bone marrow) with ${ }^{89} \mathrm{Zr}$-oxine after $7 \mathrm{~d}$ (17). Murine ${ }^{89} \mathrm{Zr}$-oxine-labeled OT-1 transgenic cytotoxic T lymphocytes (OT-1 CTLs) did not show impaired in vitro function. Labeled OT-1 CTLs $\left(7.7 \times 10^{6}\right)$ were administered to B16-OVA tumor-bearing mice. After $7 \mathrm{~d}$, labeled CTLs at the tumor site represented $5 \%$ of the whole-body activity (18). A third study with the same ${ }^{89} \mathrm{Zr}$-oxine complex focused on labeled CAR T-cell function and trafficking in vivo. Labeled interleukin 13 receptor $\alpha 2$-targeted and prostate stem cell antigen-targeted CAR T cells showed no significant loss in cytokine production, migration, or tumor cytotoxicity in vitro or antitumor activity in vivo with a load of $70 \mathrm{kBq}$ per million cells. Adoptively transferred interleukin 13 receptor $\alpha 2$-targeted CAR T cells $\left(2 \times 10^{6}\right)$ were imaged up to $6 \mathrm{~d}$ at this dose in a glioma mouse model, and $7.5 \times 10^{6}$ prostate stem cell antigen-targeted CAR T cells up to $7 \mathrm{~d}$ in a subcutaneous prostate cancer model (19). ${ }^{89} \mathrm{Zr}$-oxine has been further exemplified through labeling and imaging human $\gamma \delta \mathrm{T}$ cells (20). 


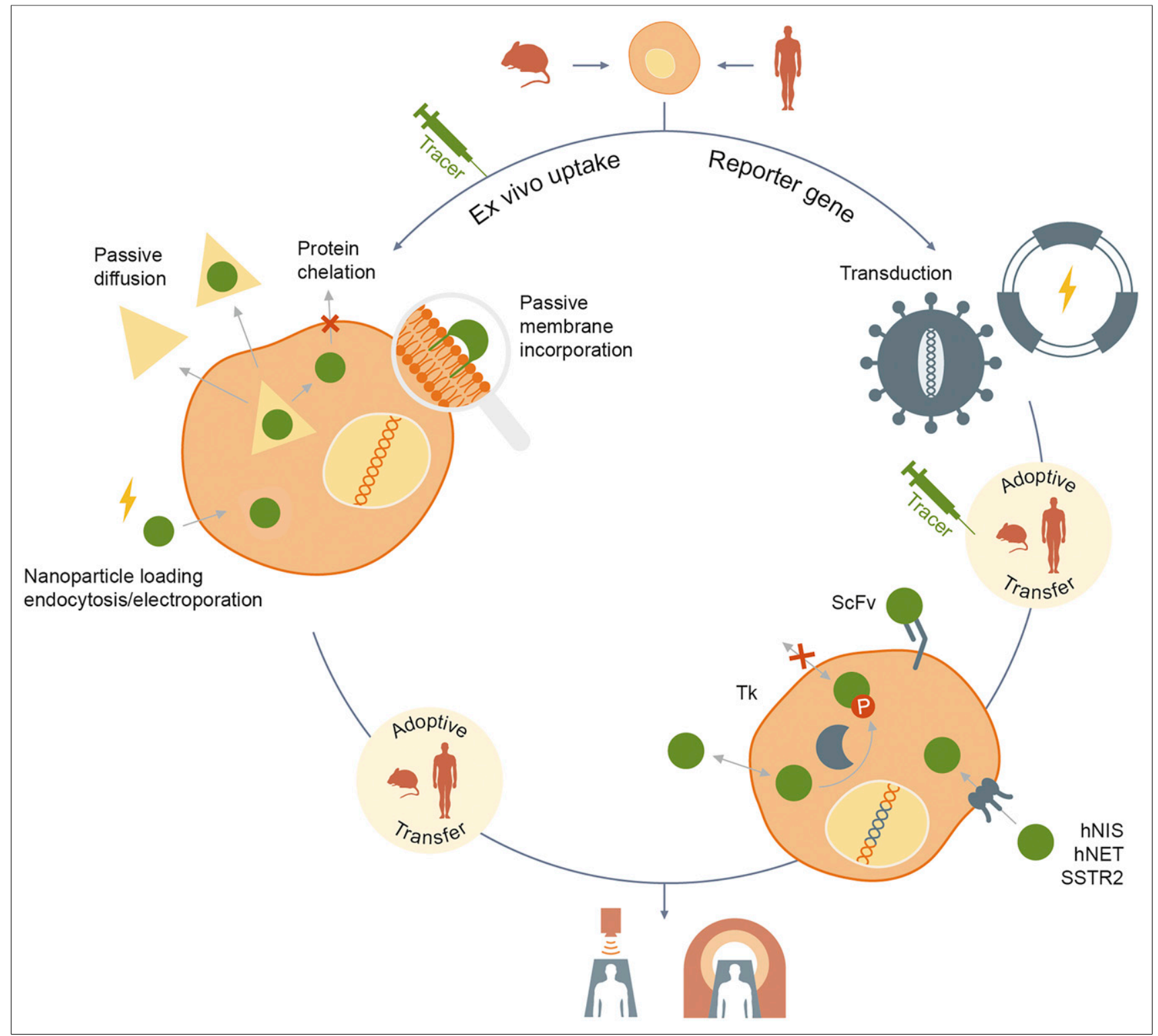

FIGURE 2. Illustration of current approaches to cell therapy imaging.

${ }^{52} \mathrm{Mn}$ ( ${ }^{52} \mathrm{Mn}$-oxine) is a PET compatible isotope with a half-life of $5.59 \mathrm{~d}$ and could extend the imaging window of passively labeled cells to $14 \mathrm{~d}$. However, even when the loading efficiency into $\gamma-\delta$-T cells was relatively good $(45.6 \% \pm 29.1 \%)$, an important efflux phenomenon was observed, with less than a third of the label maintained after $24 \mathrm{~h}(21)$.

Ultimately, even with the development of advanced labeling approaches, the passive approach to cell labeling provides only a snapshot of the behavior of cell therapies. To move the field forward and allow for imaging over the lifetime of a cell therapy or patient, we need approaches that are permanently integrated into the cell therapy for longitudinal imaging.

\section{NANOPARTICLE MRI}

SPIO-based MRI contrast agents are licensed for the imaging of liver disease (22). The high resolution delivered by these agents has great potential value. The utility of this approach is illustrated by the ex vivo SPIO labeling of a tumor cell vaccine and subsequent tracking by MRI of transfer of SPIO nanoparticles from the vaccine to dendritic cells in vivo (23). This form of nanoparticle imaging lends itself to cellular vaccine and stem cell therapy development (24).

\section{REPORTER GENE SYSTEMS FOR PRECLINICAL STUDIES}

Protocols are now well established for transducing $\mathrm{T}$ cells ex vivo to introduce the genes necessary for expression of CARs and TCRs. Vectors generally allow for the inclusion of more than one gene sequence, enabling the introduction of coexpressed reporter genes for cell imaging. Reporter gene introduction conveys advantages over passive labeling. Once the reporter gene is integrated within the T-cell genome, imaging can continue in vivo as long as the cell therapy persists. The reporter gene is passed on to daughter cells, ensuring that an expanding and contracting population can be traced. Finally, dying cells, when cleared by phagocytosis, do not pass on the reporter to macrophages. 
Bioluminescent and fluorescent reporter gene systems are widely used for imaging in preclinical settings. These approaches have successfully been adapted for the imaging of CAR T cells in vivo in tumor-bearing mice (25). Light spectrum-emitting imaging techniques have low tissue penetrance, limiting suitability for human imaging. Ultimately, this has limited incorporation of light-based imaging reporters into cell therapy research.

Both PET/CT and SPECT/CT imaging modalities are available for preclinical imaging and are also widely available for clinical imaging. This seamlessness makes translation of promising preclinical constructs into clinical trials feasible (Fig. 2).

There are a range of reporter gene systems and radiotracers available (Table 1). Radiotracers are injected into the organism and are taken up by the reporter gene-expressing cells. These injections can be repeated at multiple time points. These systems are suitable for short-half-life radioisotopes such as ${ }^{18} \mathrm{~F}$ (half-life, $110 \mathrm{~min}$ ), ${ }^{68} \mathrm{Ga}$ (half-life, $1 \mathrm{~h}$ ), or ${ }^{99} \mathrm{~m}^{\mathrm{TcO}_{4}}{ }^{-}$(half-life, $6 \mathrm{~h}$ ), limiting radiation damage. Most reporter genes require adenosine triphosphate for the active cotransport of radiotracer, with the significant benefit that only viable cells are able to take up radiotracer.

The key reporter genes that have been evaluated for imaging $\mathrm{T}$ cells in preclinical studies include the viral protein herpes simplex virus type 1 thymidine kinase (HSV1tk) and several human-derived reporters, namely the sodium iodide symporter (hNIS), norepinephrine transporter (hNET), somatostatin receptor 2, and deoxycytidine kinases (hdCK).

The HSV1tk gene has long been used as a reporter, with success in imaging donor lymphocytes in the context of allogeneic stem cell transplantation (26). One key limitation of HSV1tk is its viral origin and immunogenicity in humans. One approach to potentially overcoming this is modification of HSV1tk, replacing the nuclear localization sequence with a nuclear export signal, trapping the HSV1tk in the cytoplasm. In a study with the PET-compatible radiotracer,
$2{ }^{\prime}$ - ${ }^{18}$ F-fluoro-5-ethyl-1- $\beta$-D-arabinofuranosyluracil ( $\left.{ }^{18} \mathrm{~F}-\mathrm{FEAU}\right) \mathrm{T}$ cells were transduced with the engineered HSV1tk reporter gene and a CD19-specific CAR using the nonviral Sleeping Beauty transposon/ transposase system. The authors compared the imaging ability of their system when ${ }^{18} \mathrm{~F}$-FEAU is loaded ex vivo before subcutaneous adoptive transfer or injected in vivo $2 \mathrm{~h}$ after the subcutaneous adoptive transfer. They were able to detect $7.5 \times 10^{6} \mathrm{~T}$ cells after in vivo tracer administration. They reported a 10 -fold higher sensitivity when $\mathrm{T}$ cells are labeled ex vivo. This difference is due to the lower tissue background when the radiotracer is not injected systemically and present in the blood pool (26).

To address concerns regarding the immunogenicity of HSV1tk, human-derived reporter gene systems have been developed. The hdCK triple-mutant has an increased affinity for thymidine analogs, increasing uptake velocity at lower substrate concentrations. Hematopoietic stem cells transduced with hdCK triple-mutant can be imaged in vivo with the 1 -(2-deoxy-2-18 $\mathrm{F}$-fluoro- $\beta$-L-arabinofuranosyl)5-methyluracil probe by PET/CT (27). Coexpression of the melan-Aspecific F5 TCR with the hdCK triple-mutant was used in vivo for targeting HLA matched and mismatched melanoma cell lines. Melan-A is expressed by both the M202 and the M207 cell lines whereas M202 cells alone express HLA A*0201, which restricts peptide recognition by the F5 TCR. Preliminary in vitro studies demonstrated that reporter gene expression and function do not affect cytotoxic activity of transduced $\mathrm{T}$ cells. Animals bearing contralateral M202 and M207 xenografts were imaged at day 8 after adoptive transfer of hdCKS-F5-TCR-expressing T cells. The tumor-to-muscle signal ratio showed a 2-fold increase at the HLAmatched tumor site (28).

In a key publication in 2007, Doubrovin et al. demonstrated the utility of the hNET for imaging subcutaneously and intravenously delivered Epstein-Barr virus-specific T cells in a murine model of human Epstein-Barr virus-positive lymphoma (29). More

TABLE 1

Radionuclide Probes for Various Reporter Genes Used to Image T Cells In Vivo

\begin{tabular}{|c|c|c|c|c|}
\hline Radionuclide probe & Reporter target & Immunogenic & Endogenous expression & References \\
\hline${ }^{18} \mathrm{~F}-\mathrm{FEAU}$ & HSV1tk & Yes & None & 26,33 \\
\hline${ }^{124} \mathrm{I}-\mathrm{FIAU}$ & & & & 33 \\
\hline${ }^{18} \mathrm{~F}-\mathrm{FHBG}$ & & & & 39 \\
\hline${ }^{18} \mathrm{~F}-\mathrm{L}-\mathrm{FMAU}$ & hdCK3 & No & None & 27,28 \\
\hline${ }^{18} \mathrm{~F}-\mathrm{L}-\mathrm{FIAU},{ }^{18} \mathrm{~F}-\mathrm{FEAU}$ & hdCKDM & & None & 33 \\
\hline 123I-MIBG & hNET & No & $\begin{array}{l}\text { Neurons of sympathetic system and those } \\
\text { innervating adrenal medulla, lung and placenta }\end{array}$ & $29,30,33$ \\
\hline 124I-MIBG & & & & 29,33 \\
\hline${ }^{18} \mathrm{~F}-\mathrm{MFBG}$ & & & & 33 \\
\hline $99 \mathrm{mTcO}_{4}^{-}$ & hNIS & No & Thyroid, stomach, salivary glands & 31 \\
\hline${ }^{18} \mathrm{~F}$-tetrafluoroborate & & & & 32 \\
\hline${ }^{124}$ I-Nal & & & & 34 \\
\hline${ }^{18} \mathrm{~F}-\mathrm{NOTAOCT}$ & hSSTR2 & No & Cerebrum, kidneys, gastrointestinal tract & 35 \\
\hline${ }^{68} \mathrm{Ga}-\mathrm{DOTATOC}$ & & & & 36 \\
\hline
\end{tabular}

${ }^{124} \mathrm{I}-\mathrm{FIAU}=5$-124|-iodo-1-(2-deoxy-2-fluoro- $\beta$-D-arabinofuranosyl)uracil; ${ }^{18} \mathrm{~F}-\mathrm{FHBG}=9$ 9-[4-18F-fluoro-3-(hydroxymethyl)butyl]guanine; ${ }^{18} \mathrm{~F}-\mathrm{L}-\mathrm{FMAU}=1-\left(2-\right.$ deoxy-2-18F-fluoro- $\beta$-L-arabinofuranosyl)-5-methyluracil; ${ }^{123 / 124} \mathrm{I}-\mathrm{MIBG}=$ meta-[ ${ }^{123 / 124}$ ] ]iodobenzylguanidine; ${ }^{18} \mathrm{~F}-\mathrm{MFBG}=$ meta- $^{18} \mathrm{~F}$-fluorobenzylguanidine; ${ }^{18} \mathrm{~F}-\mathrm{NOTAOCT}={ }^{18} \mathrm{~F}-\mathrm{NOTA}$-octreotide; hdCKDM $=$ human deoxycytidine kinase double mutant; hdCK3 = human deoxycytidine kinase triple mutant; hSSTR2 = human somatostatin receptor 2. 
recently, the T-cell line Sup-T1 was engineered to express a far-red fluorescence reporter and the hNET. After intravenous administration of the hNET substrate, ${ }^{123}$ I-labeled metaiodobenzylguanidine, SPECT imaging was undertaken. After $24 \mathrm{~h}$, background signal reduced sufficiently so that specific radiotracer accumulation in subcutaneous injected hNET ${ }^{+}$Sup-T1s could be observed (30).

The hNIS has been coexpressed with a prostate-specific membrane antigen-specific CAR. No impact on cytotoxic function was seen in vitro after coculture with ${ }^{99} \mathrm{TcO}_{4}{ }^{-}$. Subcutaneous injection of $\mathrm{CAR}^{+}$ $\mathrm{T}$ cells could be imaged with a tested threshold of $0.15 \times 10^{5}$ cells by SPECT/CT. The temporal and special relationship of CAR T cells and xenografts in treatable and overwhelming tumor burden models was demonstrated with high-resolution imaging of CAR T-cell accumulation in tumor despite the natural uptake of ${ }^{99} \mathrm{TcO}_{4}{ }^{-}$via the murine NIS in stomach, salivary glands, and thyroid (Fig. 3) (31). PET imaging of hNIS with a short-half-life probe is now possible with the development of the ${ }^{18}$ F-tetrafluoroborate (32).

In a comparative study, Moroz et al. investigated the sensitivity of radiotracer PET or SPECT combinations with 4 different reporter gene systems: HSV1tk, hNET, hNIS, and hdCK double-mutant. The authors injected different doses of reporter-transduced $\mathrm{T}$ cells subcutaneously into contralateral shoulders followed by intravenous administration of the appropriate radiotracer 30 min later. At $4 \mathrm{~h}$, the $\mathrm{hNET} /{ }^{18} \mathrm{~F}-\mathrm{MFBG}$ combination exhibited the highest sensitivity due to a very low background with $1 \times 10^{5} \mathrm{~T}$ cells detected. hdCK double-mutant $/{ }^{18}$ F-FEAU and HSV1tk $/{ }^{18}$ F-FEAU both had a threshold of $3 \times 10^{5}$ transduced cells at $4 \mathrm{~h}$. The detection limit was lower with ${ }^{124}$ I radiotracer, with $1 \times 10^{6}$ transduced $\mathrm{T}$ cells the threshold for hNET at $24 \mathrm{~h}$ and hNIS at $1 \mathrm{~h}$ and $3 \times 10^{6}$ for hdCK double-mutant or HSV1tk at $4 \mathrm{~h}$ (33).

Coexpression of the SSTR2 reporter gene with anti-intercellular adhesion molecule 1 CAR followed by the administration of ${ }^{68} \mathrm{Ga}$-DOTATOC and ${ }^{18} \mathrm{~F}$-NOTA-octreotide as imaging probes has

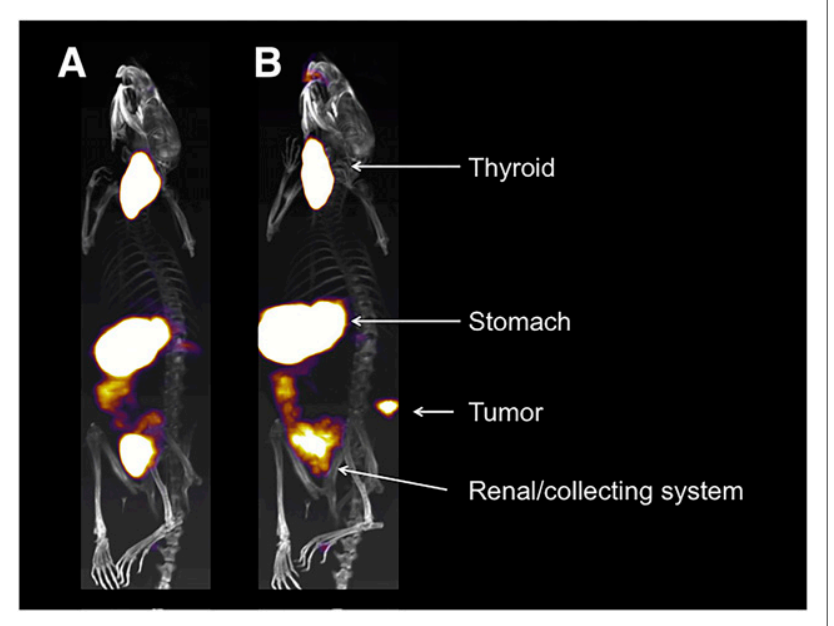

FIGURE 3. Representative SPECT/CT images of mice with flank prostate-specific membrane antigen-expressing prostate xenografts.

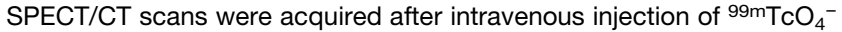
in animals $16 \mathrm{~d}$ after subcutaneous xenograft injection and $9 \mathrm{~d}$ after intravenous injection of $1 \times 10^{6} \mathrm{hNIS}$-expressing prostate-specific membrane antigen-targeting CAR T-cells with truncated CAR intracellular signaling domain (A) and fully functional CAR signaling domain (B). Physiologic uptake in thyroid and stomach and clearance of radiotracer via renal collecting system marked by white arrows. In signaling-competent CAR-treated animal, hNIS-expressing CAR T-cells are clearly visualized penetrating flank tumor. been used to image CAR T-cell infiltration into intercellular adhesion molecule 1 -positive $8505 \mathrm{C}$ tumor xenografts. The study aimed to understand why the adoptive transfer of $1.5 \times 10^{6} \mathrm{CAR}^{+}$ $\mathrm{T}$ cells at between days 7 and 10 after tumor xenograft injection led to tumor control whereas delayed adoptive transfer at days 1315 did not. By using firefly luciferase-expressing tumor cells and SSTR2-expressing CAR T cells, the authors could follow both the tumor burden and the expansion of CAR T cells in vivo (34). In the second study, the same authors engineered an anti-intercellular adhesion molecule $1 \mathrm{CAR}$ to have a range of affinities from 1.5 $\mathrm{mM}$ to $1 \mathrm{nM}$. The differing kinetics of behavior of in vivo tracked CAR T cells in xenograft-bearing mice with ${ }^{68} \mathrm{Ga}$-DOTATOC PET imaging exemplified the potential for using reporter gene imaging to investigate different cell therapy approaches (35).

In a highly innovative approach, Krebs et al. fused a single-chain fragment of an antilanthanoid-DOTA antibody to the human CD4 transmembrane domain and coexpressed with anti-CD19 CAR. CD19+-U373 tumor cells were injected subcutaneously and CAR T cells were adoptively transferred $7 \mathrm{~d}$ later, either intratumorally or subcutaneously. Thirty minutes after adoptive transfer, the lanthanoid probes ${ }^{86}$ Y-lanthanoid or ${ }^{177} \mathrm{Lu}$-lanthanoid were injected intravenously to enable PET and SPECT imaging, respectively. The lanthanoid probes bind irreversibly to the single-chain fragment. Unbound probe is rapidly cleared. Signal-to-background ratio was maximal after $16 \mathrm{~h}$ for PET imaging and $4 \mathrm{~h}$ for SPECT (36).

\section{CLINICAL DEVELOPMENT}

The next step in developing ex vivo and reporter gene imaging for cell therapy is noninvasive human imaging. One patient treated with ex vivo expanded peptide vaccine-boosted tumor-infiltrating lymphocyte therapy for HER $2^{+}$breast cancer received ${ }^{111} \mathrm{In}$-labeled cells and underwent SPECT/CT imaging. The 24- to 48-h images show T-cell migration to sites of known bony metastatic disease (37). Keu et al. have led the field in demonstrating the utility of reporter gene imaging in human studies. They have coexpressed the HSV1tk with an interleukin-13 receptor-directed CAR. Seven patients have been treated, and the fate of the infused cells has been monitored by PET imaging after regional delivery into the surgical bed of debulked intracerebral glioma. This work demonstrates the clear clinical utility of reporter gene imaging in clinical trials of cell therapies. Clinical efficacy of the interleukin-13 receptor-directed therapy in a single patient was published previously by the group (38); however, the authors acknowledge that the linking of imaging signal to clinical outcomes and to physical assessment of cell trafficking and survival was not possible in this study. This represents a limitation of the published work (39).

Ex vivo SPIO-labeled dendritic cells have been delivered by coinjection in human melanoma trials of dendritic cell vaccination (40). The impact of this approach in the clinic is currently limited by the disappointing performance of dendritic cell vaccination as a clinical modality. Stem cell therapy and its potential compatibility with SPIO labeling may lead to developments in this area in human studies.

\section{CONCLUSION}

Preclinical development of cell therapy imaging approaches has advanced to the point that incorporation into clinical studies is a real and present capability. Multiple approaches are in development. Further work is needed to understand the kinetics, in humans, of radiotracers and reporters in dynamic cell-based imaging studies to allow for absolute quantitation of cell number on expansion and 
contraction in regions of interest. Ultimately, incorporating imaging at the point of clinical translation will allow noninvasive endpoints to aid in the selection of promising cell-based therapies for human disease.

\section{DISCLOSURE}

Research in the authors' laboratories is supported by the Medical Research Council, Cancer Research U.K., philanthropic funding from Mr. J Reece, Bayer HealthCare, Bloodwise, the King's Health Partners Research and Development Fund, the British Council Newton Fund Institutional Links Award, the British Lung Foundation, Breast Cancer Now, the Experimental Cancer Medicine Centre at King's College London, the King's Health Partners Cancer Research U.K. Cancer Centre, the National Institute for Health Research (NIHR) Biomedical Research Centre based at Guy's and St Thomas' NHS Foundation Trust and King's College London, the Cancer Research U.K. Barts Centre, the Barts Charity, Invicro LLC, and the National Centre for the Replacement, Refinement and Reduction of Animals in Research (NC3Rs). The views expressed are those of the authors only. John Maher is chief scientific officer of Leucid Bio, a spinout company focused on CAR T-cell immunotherapies for malignant disease. No other potential conflict of interest relevant to this article was reported.

\section{ACKNOWLEDGMENTS}

We thank Florian Casarin and Flème Studio for assistance with graphic design.

\section{REFERENCES}

1. Maldini CR, Ellis GI, Riley JL. CAR T cells for infection, autoimmunity and allotransplantation. Nat Rev Immunol. 2018;18:605-616.

2. Kochenderfer JN, Dudley ME, Kassim SH, et al. Chemotherapy-refractory diffuse large B-cell lymphoma and indolent B-cell malignancies can be effectively treated with autologous $\mathrm{T}$ cells expressing an anti-CD19 chimeric antigen receptor. J Clin Oncol. 2015;33:540-549.

3. Maude SL, Frey N, Shaw PA, et al. Chimeric antigen receptor T cells for sustained remissions in leukemia. N Engl J Med. 2014;371:1507-1517.

4. Morgan RA, Chinnasamy N, Abate-Daga D, et al. Cancer regression and neurological toxicity following anti-MAGE-A3 TCR gene therapy. I Immunother. 2013;36:133-151.

5. Robbins PF, Morgan RA, Feldman SA, et al. Tumor regression in patients with metastatic synovial cell sarcoma and melanoma using genetically engineered lymphocytes reactive with NY-ESO-1. J Clin Oncol. 2011;29:917-924.

6. Dudley ME, Yang JC, Sherry R, et al. Adoptive cell therapy for patients with metastatic melanoma: evaluation of intensive myeloablative chemoradiation preparative regimens. J Clin Oncol. 2008;26:5233-5239.

7. Radvanyi LG, Bernatchez C, Zhang M, et al. Specific lymphocyte subsets predict response to adoptive cell therapy using expanded autologous tumor-infiltrating lymphocytes in metastatic melanoma patients. Clin Cancer Res. 2012;18:6758-6770.

8. Brentjens R, Yeh R, Bernal Y, Riviere I, Sadelain M. Treatment of chronic lymphocytic leukemia with genetically targeted autologous $\mathrm{T}$ cells: case report of an unforeseen adverse event in a phase I clinical trial. Mol Ther. 2010;18:666-668.

9. Lamers $\mathrm{CH}$, Sleijfer S, Vulto AG, et al. Treatment of metastatic renal cell carcinoma with autologous T-lymphocytes genetically retargeted against carbonic anhydrase IX: first clinical experience. J Clin Oncol. 2006;24:e20-e22.

10. Morgan RA, Yang JC, Kitano M, Dudley ME, Laurencot CM, Rosenberg SA. Case report of a serious adverse event following the administration of $\mathrm{T}$ cells transduced with a chimeric antigen receptor recognizing ERBB2. Mol Ther. 2010;18:843-851.

11. Neelapu SS, Tummala S, Kebriaei P, et al. Chimeric antigen receptor T-cell therapy: assessment and management of toxicities. Nat Rev Clin Oncol. 2018;15: 47-62.

12. Malviya G, Galli F, Sonni I, Signore A. Imaging T-lymphocytes in inflammatory diseases: a nuclear medicine approach. Q J Nucl Med Mol Imaging. 2014;58: $237-257$.
13. Youniss FM, Sundaresan G, Graham LJ, et al. Near-infrared imaging of adoptive immune cell therapy in breast cancer model using cell membrane labeling. PLoS One. 2014;9:e109162.

14. Bhatnagar P, Li Z, Choi Y, et al. Imaging of genetically engineered $\mathrm{T}$ cells by PET using gold nanoparticles complexed to copper-64. Integr Biol (Camb). 2013;5:231-238.

15. Bhatnagar P, Alauddin M, Bankson JA, et al. Tumor lysing genetically engineered T cells loaded with multi-modal imaging agents. Sci Rep. 2014;4:4502.

16. Meir R, Shamalov K, Betzer O, et al. Nanomedicine for cancer immunotherapy: tracking cancer-specific T-Cells in vivo with gold nanoparticles and CT imaging. ACS Nano. 2015;9:6363-6372.

17. Charoenphun P, Meszaros LK, Chuamsaamarkkee K, et al. $\left[{ }^{89} \mathrm{Zr}\right]$ oxinate4 for long-term in vivo cell tracking by positron emission tomography. Eur J Nucl Med Mol Imaging. 2015;42:278-287.

18. Sato N, Wu H, Asiedu KO, Szajek LP, Griffiths GL, Choyke PL. ${ }^{89} \mathrm{Zr}$-oxine complex PET cell imaging in monitoring cell-based therapies. Radiology. 2015; 275:490-500.

19. Weist MR, Starr R, Aguilar B, et al. PET of adoptively transferred chimeric antigen receptor T cells with ${ }^{89} \mathrm{Zr}$-oxine. J Nucl Med. 2018;59:1531-1537.

20. Man F, Lim L, Volpe A, et al. In vivo PET tracking of ${ }^{89} \mathrm{Zr}$-labeled Vy ${ }^{\mathrm{s} \delta} 2 \mathrm{~T}$ cells to mouse xenograft breast tumors activated with liposomal alendronate. $\mathrm{Mol}$ Ther. 2019;27:219-229.

21. Gawne P, Man F, Fonslet J, et al. Manganese-52: applications in cell radiolabelling and liposomal nanomedicine PET imaging using oxine (8-hydroxyquinoline) as an ionophore. Dalton Trans. 2018;47:9283-9293.

22. Wang YX. Superparamagnetic iron oxide based MRI contrast agents: current status of clinical application. Quant Imaging Med Surg. 2011;1:35-40.

23. Long CM, van Laarhoven HW, Bulte JW, Levitsky HI. Magnetovaccination as a novel method to assess and quantify dendritic cell tumor antigen capture and delivery to lymph nodes. Cancer Res. 2009;69:3180-3187.

24. Bulte JW. In vivo MRI cell tracking: clinical studies. AJR. 2009;193:314-325.

25. Santos EB, Yeh R, Lee $J$, et al. Sensitive in vivo imaging of $\mathrm{T}$ cells using a membrane-bound Gaussia princeps luciferase. Nat Med. 2009;15:338-344.

26. Bonini C, Ferrari G, Verzeletti S, et al. HSV-TK gene transfer into donor lymphocytes for control of allogeneic graft-versus-leukemia. Science. 1997;276: 1719-1724.

27. McCracken MN, Gschweng EH, Nair-Gill E, et al. Long-term in vivo monitoring of mouse and human hematopoietic stem cell engraftment with a human positron emission tomography reporter gene. Proc Natl Acad Sci USA. 2013;110:1857-1862.

28. McCracken MN, Vatakis DN, Dixit D, McLaughlin J, Zack JA, Witte ON. Noninvasive detection of tumor-infiltrating T cells by PET reporter imaging. J Clin Invest. 2015;125:1815-1826.

29. Doubrovin MM, Doubrovina ES, Zanzonico P, Sadelain M, Larson SM, O'Reilly RJ. In vivo imaging and quantitation of adoptively transferred human antigen-specific $\mathrm{T}$ cells transduced to express a human norepinephrine transporter gene. Cancer Res. 2007;67:11959-11969.

30. Badar A, Kiru L, Kalber TL, et al. Fluorescence-guided development of a tricistronic vector encoding bimodal optical and nuclear genetic reporters for in vivo cellular imaging. EJNMMI Res. 2015;5:18.

31. Emami-Shahri N, Foster J, Kashani R, et al. Clinically compliant spatial and temporal imaging of chimeric antigen receptor T-cells. Nat Commun. 2018;9:1081.

32. O'Doherty J, Jauregui-Osoro M, Brothwood T, et al. ${ }^{18} \mathrm{~F}$-tetrafluoroborate, a PET probe for imaging sodium/iodide symporter expression: whole-body biodistribution, safety, and radiation dosimetry in thyroid cancer patients. $\mathrm{J} \mathrm{Nucl} \mathrm{Med}$. 2017;58:1666-1671.

33. Moroz MA, Zhang H, Lee J, et al. Comparative analysis of T cell imaging with human nuclear reporter genes. J Nucl Med. 2015;56:1055-1060.

34. Vedvyas Y, Shevlin E, Zaman M, et al. Longitudinal PET imaging demonstrates biphasic CAR T cell responses in survivors. JCI Insight. 2016;1:e90064.

35. Park S, Shevlin E, Vedvyas Y, et al. Micromolar affinity CAR T cells to ICAM-1 achieves rapid tumor elimination while avoiding systemic toxicity. Sci Rep. 2017;7:14366.

36. Krebs S, Ahad A, Carter L, et al. Antibody with infinite affinity for in vivo tracking of genetically engineered lymphocytes. J Nucl Med. 2018;59:1894-1900.

37. Stanton SE, Eary JF, Marzbani EA, et al. Concurrent SPECT/PET-CT imaging as a method for tracking adoptively transferred T-cells in vivo. J Immunother Cancer. 2016;4:27.

38. Brown CE, Alizadeh D, Starr R, et al. Regression of glioblastoma after chimeric antigen receptor T-cell therapy. N Engl J Med. 2016;375:2561-2569.

39. Keu KV, Witney TH, Yaghoubi S, et al. Reporter gene imaging of targeted T cell immunotherapy in recurrent glioma. Sci Transl Med. 2017;9(373):eaag2196.

40. de Vries IJ, Lesterhuis WJ, Barentsz JO, et al. Magnetic resonance tracking of dendritic cells in melanoma patients for monitoring of cellular therapy. Nat Biotechnol. 2005;23:1407-1413. 